\title{
Combination of Percutaneous Lung Biopsy and Xpert MTB/RIF Ultra Enhances the Differential Diagnosis of Tuberculosis: A Prospective Cohort Study
}

\author{
Zhen Zhou · Chenghai Li · Rui Zhu • Dongpo Wang • \\ Tao Liu · Junnan Jia · Fen Wang · Liping Zhao · Lingling Dong • \\ Xia Yu $\cdot$ Hairong Huang
}

Received: July 4, 2020 / Published online: August 8, 2020

(C) The Author(s) 2020

\begin{abstract}
Introduction: To evaluate the value of the combination of computed tomography (CT)guided percutaneous lung biopsy and Xpert MTB/RIF Ultra (Xpert-Ultra) in enhancing the differential diagnosis of tuberculosis.
\end{abstract}

Zhen Zhou, Chenghai Li and Rui Zhu contributed equally to this study.

Digital Features To view digital features for this article go to https://doi.org/10.6084/m9.figshare.12739286.

Z. Zhou · C. Li · D. Wang

Department of Radiology, Beijing Chest Hospital, Capital Medical University, Beijing Tuberculosis and Thoracic Tumor Institute, Beijing, China

R. Zhu · J. Jia · F. Wang · L. Zhao · L. Dong ·

$\mathrm{X} . \mathrm{Yu}(\square) \cdot \mathrm{H}$. Huang $(\square)$

National Tuberculosis Clinical Laboratory, Beijing

Key Laboratory for Drug Resistance Tuberculosis Research. Beijing Chest Hospital, Capital Medical University, Beijing Tuberculosis and Thoracic

Tumor Institute, Beijing, China

e-mail: yuxiasmart@163.com

H. Huang

e-mail: huanghairong@tb123.org

T. Liu

Department of Anesthesiology, Beijing Chest

Hospital, Capital Medical University, Beijing

Tuberculosis and Thoracic Tumor Institute, Beijing, China
Methods: Patients with lesions in the lungs for whom the differential diagnosis was difficult were prospectively and consecutively enrolled. Specimens collected by percutaneous lung biopsy were subject to Xpert-Ultra and histopathologic examination, respectively.

Results: Biopsy was successfully performed for 147 patients who were eligible for analysis, including $65 \mathrm{~TB}, 55$ lung cancer and 27 other chronic infection cases. The sensitivity, specificity, positive predictive value and negative predictive value of Xpert-Ultra for TB diagnosis were $75.38 \%(49 / 65)$ and $95.12 \%$ (78/82), $92.45 \%$ (49/53) and $82.98 \%$ (78/94), respectively. Among patients with strong evidence for TB diagnosis (categorized as confirmed or probable TB cases), the overall positive rate of the Xpert-Ultra assay was $83.63 \%$ (46/55), which was much higher than for any reported sample type with negative smear test outcomes to date. Five rifampicin (RIF)-resistant cases were identified using the biopsy tissues. However, "trace" positive did not seem reliable for TB diagnosis with lung biopsy specimens; a $25.61 \%(21 / 82)$ "trace"-positive rate was acquired in the non-TB group.

Conclusion: Percutaneous lung biopsy combined with Xpert-Ultra produced high sensitivity for culture-negative pulmonary TB patient diagnosis. "Trace" outcome might not be a reliable positive category for lung biopsy specimens. 
Trial registration: ClinicalTrials.gov identifier, ChiCTR1900026412. Retrospectively registered on Oct 8th, 2019).

Keywords: Differential diagnosis; Percutaneous lung biopsy; Tuberculosis; Xpert MTB/RIF Ultra

\section{Key Summary Points}

Percutaneous lung biopsy combined with Xpert-Ultra produced high sensitivity for culture-negative tuberculosis patient diagnosis and rapid rifampicin-resistant detection.

Xpert-Ultra with a "trace" positive outcome did not seem reliable for the lung biopsy specimens.

Percutaneous lung biopsy combined with Xpert-Ultra facilitates tuberculosis diagnosis and can be used for differential diagnosis of lung disease.

\section{INTRODUCTION}

Tuberculosis (TB) is the second most prevalent infectious disease in China with $\sim 0.866$ million new cases reported in 2019 [1]. Lung cancer is the most common cancer type and the leading cause of cancer-related deaths in China. In 2018, 0.77 million new cases of lung cancer and 0.69 million incurred deaths were reported [2]. The increasing percentage of the aging population will result in a continuous increase in the prevalence of lung cancer.

The simultaneous high prevalence of $\mathrm{TB}$ and lung cancer requires an effective differential diagnosis between these two diseases, which is currently an enormous challenge for respiratory physicians. Although multiple new and increasingly sensitive tests have been applied for TB diagnosis, the acid fast bacilli (AFB)-positive rate among $\mathrm{TB}$ patients remains low. According to the WHO's 2019 Global TB Report, only $37 \%$ of the TB patients in China presented bacteriologic evidence despite considering all the available outcomes of conventional techniques and new molecular testing. In addition, TB often appears similar to lung cancer on $\mathrm{X}$-rays and often mimics malignancy on positron emission tomography (PET)/computed tomography (CT) as well. False positives are not rare for TB lesions when a standard uptake value $(\mathrm{SUV})>2.5$ is used as the cutoff for differentiating lung malignancies from other benign diseases using F-18 fluoro-D-glucose (FDG) PET/ CT [3]. Meanwhile, less invasive examination for lung cancer, e.g., detection of tumor biomarkers, is often not very sensitive and has low specificity [4]. Therefore, pulmonary doctors often encounter difficulty when making the differential diagnosis for patients with abnormal pulmonary images.

CT-guided percutaneous lung biopsy has commonly been used because of its application in the highly lethal nature of lung cancer [5]. Xpert MTB/RIF Ultra (Xpert-Ultra) is one of the most sensitive methods for TB diagnosis right now [6]. It can successfully detect Mycobacterium tuberculosis (MTB) with as low as $13 \mathrm{cfu} / \mathrm{ml}$ AFB load. Studies have reported that Xpert-Ultra could find more positive cases among culturenegative cases [7]. In this study, we combined CT-guided percutaneous lung biopsy with Xpert MTB/RIF Ultra to evaluate its rapid and explicit TB diagnosis value in differentiating lung lesions.

\section{METHODS}

\section{Ethics Approval}

The study was approved by the Ethics Committee of the Beijing Chest Hospital, Capital Medical University (YJS-2019008). The study was conducted according to the Helsinki Declaration of 1964 , as revised in 2013, concerning human and animal rights. Written informed consent was obtained from all the participants. The trial was retrospectively registered at www. clinicaltrials.gov (ChiCTR1900026412). 


\section{Study Design}

The biopsy tissue was obtained by CT-guided percutaneous core-needle lung biopsy. Patients who had all types of lesions in the lung and posed difficulties in the differential diagnosis were prospectively and consecutively enrolled from August 2019 to January 2020 in Beijing Chest Hospital (Beijing, China). Specimens collected from each patient during operations were respectively subjected to Xpert-Ultra and histopathologic examination. For patient who could not be explicitly diagnosed with the biopsy examination, their next medical interventions were followed up until a clear diagnosis was made.

\section{Patient Enrollment}

\section{Inclusion Criteria:}

(1) 15-90 years old;

(2) Mass lesion detected on the CT image of the lung and lesion size $\geq 5 \mathrm{~mm}$ without signs of calcification;

(3) All conventional and molecular tests with sputa ( $\geq 3$ smear tests, $\geq 2$ cultures and $\geq 2$ Xpert assays) for tuberculosis diagnosis produced negative outcomes;

(4) All conventional and molecular tests with broncheoalveolar lavage fluid (BALF) and biopsy tissue ( $\geq 1$ smear test, $\geq 1$ culture and $\geq 1$ Xpert assays) collected by bronchoscopy produced negative outcomes.

(5) Trial of anti-inflammatory treatment for 2 weeks using broad-spectrum antibiotics, such as cephalosporin or fluoroquinolone, did not lead to obvious improvement.

(6) The patient understood the nature of the study and gave informed consent.

\section{Exclusion Criteria:}

(1) Uncontrollable cough or other conditions impeding biopsy.

(2) Bleeding tendency or coagulation dysfunction.

(3) Severe cardiopulmonary insufficiency, e.g., severe pulmonary hypertension.

(4) Mental disorder.
(5) Post-pneumonectomy.

\section{CT-Guided Percutaneous Lung Biopsy}

Biopsy was performed by radiologic professionals at our Radiology Department. According to the routine data, the success rate of acquiring tissue from the target lesions by CT-guided percutaneous lung biopsy was $>95 \%$, whereas the chance of adverse events that required further medical intervention was about 6-7\%. A GE 64 VCT scanner (General Electric, USA, Japan subdivision) was used to guide the biopsy. The patient was positioned with the affected side up in the lateral decubitus position. Under moderate sedation, using fentanyl and midazolam, the patient was locally anesthetized with $2 \%$ lidocaine followed by making a small incision at the planned site of entry. A $17 \mathrm{G}$ core needle (Argon, USA) was used for biopsy. One pass was made, and three biopsy tissue specimens were collected, two for histologic examination and one for Xpert-Ultra assay.

\section{Pathologic Examination}

The tissue specimens were fixed in neutral formalin, dehydrated and subsequently paraffinembedded. The $4-\mu \mathrm{m}$ sections were stained with hematoxylin and eosin solution and observed by light microscopy for patho-morphologic changes. The AFB test and/or TB-DNA PCR test was performed with tissue as required by the pathologists. Briefly, $4.0-\mu \mathrm{m}$ sections were dewaxed by dimethyl benzene and then sequentially washed with 95\%, 90\%, 85\% and $70 \%$ ethanol and finally by de-ionized water. After drying completely, the slides were stained by the standard Zeihl-Neelsen method, and AFB was detected under an oil immersion lens ( $\times$ 1000). TB-DNA PCR was performed with the MTB DNA detection kit (Daan Gene Ltd., Beijing, China).

\section{Patient Categories}

Patient categories were defined according to the following diagnostic criteria: (1) confirmed TB: 
granulomatous inflammation, with or without caseous necrosis, was observed by pathologic examination; AFB was observed in the biopsy tissue and/or TB-DNA was detected by PCR; (2) probable TB: typical chronic granulomatous inflammation with caseous necrosis was observed, and the AFB smear test and PCR-TB test were both negative if performed; (3) possible TB: non-typical chronic granulomatous inflammation or caseous necrosis were observed, and the AFB smear test and PCR-TB test were both negative if performed, with no available evidence supporting other diseases; (4) non-TB: cancer or other diseases, diagnosed by histopathologic examination using the biopsy sample or other tests.

\section{Xpert-Ultra Assay}

The tissue sample was collected in a sterilized Eppendorf tube and used for the test within a few hours. The specimen was frozen if it could not be tested in time. The biopsy tissue was homogenized using a MP FastPrep-24 homogenizer (MP Co., USA), the homogenate was then mixed with $2 \mathrm{ml}$ sample reagent, and the XpertUltra assay was performed according to the manufacturer's instructions. Seven "normal" tissue specimens, inspected visually, were collected from lung resection specimens of lung cancer patients. Xpert-Ultra was used for these specimens to evaluate the "trace" outcome.

\section{Data Management}

SPSS version 19.0 (IBM, Armonk, NY, USA) was used to compare baseline clinical characteristics, and the demographic data were analyzed via the Mann-Whitney $U$ test for continuous variables and chi-squared test for categorical variables. The sensitivity, specificity, positive predictive value (PPV) and negative predictive value (NPV) according to Xpert-Ultra were calculated against the reference standard.

\section{RESULTS}

\section{Patient Enrollment}

In total, 169 patients were enrolled (Fig. 1). Twelve quit before the operation because of concerns about operation-associated risks. Percutaneous biopsy was successfully performed for 157 patients. Thirty-five (22.29\%) patients experienced adverse events, and eight (5.10\%) of them required specific medical interventions. Pneumothorax occurred in 22 patients, but only 5 of them needed thoracic close drainage because the volume of the same lateral lung condensed $>20 \%$. The remaining 17 patients recovered without any specific medical intervention. Bleeding occurred in nine patients. However, only three of them with hemoptysis received hemostatic treatment. Furthermore, two patients suffered a small volume of bleeding in the thorax. Since no symptom was caused, no specific action was taken for them. The blood was then absorbed gradually under cautious medical supervision. Two patients also had percutaneous emphysema, which did not require any treatment. After excluding five patients with undetermined Ultra outcomes (error) and 5 defaulted patients, 147 patients were recruited for the final analysis. The median age of the enrolled patients was $50.79 \pm 17.26$ (range 14-81) years, 67.35\% (99/147) were male, and $15.65 \%(23 / 147)$ had a prior history of tuberculosis. All patients were HIV-negative. The TB patients were generally younger and had fewer underlying diseases than the non-TB patients. Basic characteristics of the enrolled patients are shown in Table 1.

\section{Pathologic Examination Outcomes}

Tissues of the target lung lesions were successfully obtained from the enrolled patients. Among them, AFB was detected in 23 specimens whereas 41 were TB-DNA positive. Three AFBpositive specimens produced negative TB-DNA outcomes, while two were identified as $M$. intracellulare by the molecular method. These two $M$. intracellulare infection cases were categorized into the non-TB group for further 


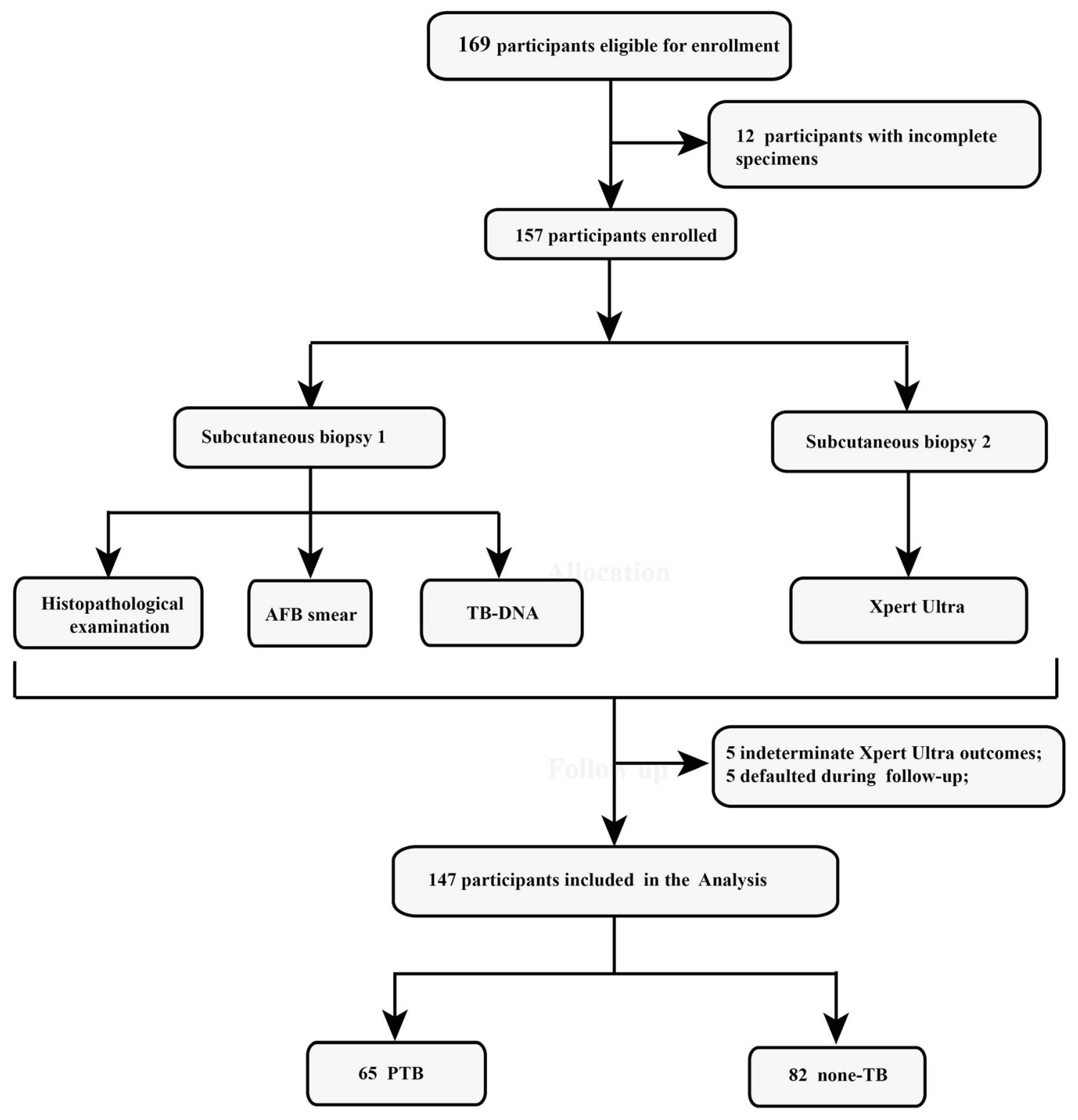

Fig. 1 Flow chart indicating the process of patient enrollment

analysis. According to the outcomes of the pathologic examination, AFB smear test and TBDNA testing, 42 patients were defined as having confirmed PTB, 13 as having probable TB and 10 as having possible TB. Among the 82 non-TB cases, 55 were diagnosed as having lung cancer according to the outcomes of cytologic examination, whereas 27 were identified as having chronic infectious diseases. Furthermore, four cases were diagnosed as having lung cancer with TB comorbidity. Since these patients did not present bacteriologic evidence but typical TB pathologic granulomatous, they were categorized into the probable $\mathrm{TB}$ group for further analysis.

\section{Xpert-Ultra Outcomes}

The Xpert-Ultra outcomes of the enrolled patients are provided in Table 2. One of the seven "normal" lung tissues collected from the 
Table 1 Demographic and clinical characteristics of the study participants

\begin{tabular}{lllr}
\hline Characteristics & TB $(\boldsymbol{n}=\mathbf{6 5})$ & Non-TB $(\boldsymbol{n}=\mathbf{8 2})$ & $\boldsymbol{p}$ value \\
\hline Gender (male/female) & $48 / 17$ & $51 / 31$ & 0.135 \\
Age, median (range), years & $43.88 \pm 18.44(17-81)$ & $56.27 \pm 14.13(14-80)$ & $<0.001$ \\
Distance from pleura (mm) & $15.52 \pm 12.63$ & $20.69 \pm 13.37$ & 0.019 \\
Nodule diameter (mm) & $30.05 \pm 16.08$ & $45.67 \pm 26.81$ & $<0.001$ \\
Initial symptoms & & & 0.198 \\
Fever, $n$ (\%) & $12(18.46)$ & $9(13.84)$ & 0.276 \\
Cough, $n$ (\%) & $29(44.61)$ & $44(67.69)$ & 0.283 \\
Pectoralgia, $n$ (\%) & $13(20.00)$ & $11(16.92)$ & 0.557 \\
Expectoration, $n(\%)$ & $29(44.61)$ & 0.978 \\
Hemoptysis, $n$ (\%) & $20(30.76)$ & $5(7.69)$ & 0.080 \\
History of tuberculosis & $3(4.61)$ & $9(13.84)$ & \\
Underlying diseases & $14(21.53)$ & & 0.704 \\
Diabetes mellitus, $n(\%)$ & $11(16.92)$ & $12(18.46)$ & $<001$ \\
Hypertension, $n$ (\%) & $6(9.23)$ & $28(43.07)$ & 0.019 \\
Kidney disease, $n(\%)$ & $3(4.61)$ & $14(21.53)$ & 0.012 \\
Hypoproteinemia, $n(\%)$ & $3(4.61)$ & $15(23.07)$ & 0.305 \\
Hydrothorax, $n$ (\%) & $2(3.07)$ & $7(10.76)$ & \\
\hline
\end{tabular}

Table 2 Xpert-Ultra outcomes of the 147 enrolled cases

\begin{tabular}{|c|c|c|c|c|c|}
\hline \multirow{2}{*}{$\begin{array}{l}\text { Xpert-Ultra } \\
\text { outcome }\end{array}$} & \multicolumn{3}{|c|}{ Tuberculosis (65) } & \multicolumn{2}{|l|}{ Non-TB (82) } \\
\hline & $\begin{array}{l}\text { Confirmed TB } \\
(42)\end{array}$ & $\begin{array}{l}\text { Probable TB } \\
(13)^{\mathbf{b}}\end{array}$ & $\begin{array}{l}\text { Possible TB } \\
\text { (10) }\end{array}$ & $\begin{array}{l}\text { Lung cancer } \\
(55)\end{array}$ & $\begin{array}{l}\text { Non-TB infection } \\
(27)^{\mathrm{a}}\end{array}$ \\
\hline Negative & 1 & 1 & 3 & 39 & 18 \\
\hline Trace & 5 & 2 & 4 & 15 & 6 \\
\hline Very low & 8 & 5 & 1 & 1 & 2 \\
\hline Low & 19 & 5 & 2 & 0 & 1 \\
\hline Medium & 6 & 0 & 0 & 0 & 0 \\
\hline High & 3 & 0 & 0 & 0 & 0 \\
\hline
\end{tabular}

a Two cases with $M$. intracellulare infection were categorized into the non-TB infection group

b Four cases diagnosed with lung cancer with TB comorbidity were categorized into the "probable TB" group for analysis 
Table 3 Performance of Xpert-Ultra when considering "trace" outcome differently

\begin{tabular}{lllc}
\hline Criteria & Xpert-Ultra outcome & TB (65) & Non-TB (82) \\
\hline Trace as negative & Positive & 49 & 4 \\
& Negative & 16 & 78 \\
Trace as positive & Positive & 60 & 25 \\
& Negative & 5 & 57 \\
\hline
\end{tabular}

lung resection specimens of lung cancer patients produced "trace" outcomes, while the other six were all negative.

Two independent criteria were applied to evaluate the performance of Xpert-Ultra (Table3): (1) considering "trace" as negative outcome: the sensitivity, specificity, PPV and NPV of Xpert-Ultra for TB diagnosis were $75.38 \%$ (49/65), 95.12\% (78/82), 92.45\% (49/ 53 ) and $82.98 \%$ (78/94), respectively; (2) considering "trace" as positive outcome: the sensitivity, specificity, PPV and NPV of Xpert-Ultra for TB diagnosis were $92.31 \%(60 / 65), 69.51 \%$ $(57 / 82), 70.59 \%(60 / 85)$ and $91.94 \%(57 / 62)$, respectively.

\section{RIF Resistance Detection}

In total, six specimens yielded RIF-resistance outcomes, including one with "high" level positive, four with "low" level positive and one with "very low" positive outcomes. RIF-resistant outcomes with "very low" positive levels are known to be unreliable [8]; therefore, this category was not adopted as resistance in this study.

\section{DISCUSSION}

The diagnosis of paucibacillary TB is challenging, even using the Xpert assay, which is one of the most sensitive methods for TB diagnosis [9]. Non-invasive imagologic examinations, such as radiologic examination or ultrasound, are mainstays for bacteriologic-negative TB diagnosis, whereas their specificities are often suboptimal $[10,11]$. Xpert-Ultra was developed to improve the sensitivity for detection of paucibacillary TB and has been successfully used for different types of clinical specimens [12, 13]. Percutaneous biopsy has been used for diagnosis of lung cancer, and its value has been validated in multiple other studies and in this study as well [14]. In our study, 59 patients were diagnosed with lung cancer according to the cytologic examinations with the biopsy tissues. However, the combination of percutaneous lung biopsy and Xpert MTB/RIF Ultra for rapid TB diagnosis had not been evaluated before.

In contrast with the first-generation Xpert, an additional semi-quantitative category of "trace" was introduced for Ultra, which indicated successful amplification of the IS6110/ IS1081 sequence, but not the $r p o B$ gene, for identification of paucibacillary samples [6]. The rationale behind this design was that there are multiple copies of the IS6110/IS1081 sequence but only a single copy of the $r p o B$ gene in the MTB genomes. Although this design improved the sensitivity of the Xpert assay, a significant number of false-positive outcomes were identified using modeling studies and on-site evaluations considering the "trace" outcomes, especially in high-TB-prevalence settings $[15,16]$. Dorman et al. recommended a conditional trace approach (a trace-positive result was reclassified as tuberculosis-negative for patients with a history of tuberculosis) or a trace-repeat approach (a trace-positive result was classified as tuberculosis-negative when a repeat Ultra test produced a negative outcome, or as tuberculosis-positive when positive), and both approaches improved Ultra's specificity and retained most of its sensitivity in smear-negative sputa [17]. We obtained a $16.92 \%(11 / 65)$ and $25.61 \%$ $(21 / 82)$ "trace"-positive rate in TB and non-TB 
groups, respectively. The surprisingly high percentage of trace outcomes in the non-TB group cannot be simply explained by the high burden of TB in the country, TB history or concealed TB. Although three out of nine non-TB patients with prior TB histories produced "trace" outcomes, many other non-TB patients with "trace" outcomes did not have a known TB history (data not shown). The underlying reason requires more investigation. We tested seven "normal" lung tissue specimens collected from lung cancer patients by visual inspection, and one of them, despite no prior TB history, produced a "trace" outcome. Therefore, we presume that "trace" outcome might not be a reliable positive category for a lung biopsy specimen in a high-TB-prevalence setting. WHO deemed thata trace-positive result is more reliable for extrapulmonary TB than for pulmonary TB patients and recommended a repeat test to avoid misleading outcomes [18]. Due to the extremely small volume of biopsy tissue in this study, we could not repeat the testing in this assay.

According to this study, the percutaneous biopsy tissue worked very well for TB detection using Xpert-Ultra. Since Xpert-Ultra can be done within a few hours, it enables a rapid differential diagnosis. Despite very low volumes of biopsy tissue, high positive rates were acquired, i.e., $85.71 \% \quad(36 / 42), \quad 76.92 \% \quad(10 / 13)$ and $30.00 \%(3 / 10)$ in patient groups with confirmed $\mathrm{TB}$, probable $\mathrm{TB}$ and possible $\mathrm{TB}$, respectively. Among patients with strong evidence for $\mathrm{TB}$ diagnosis (confirmed $\mathrm{TB}$ and probable $\mathrm{TB}$ groups), the overall positive rate was $83.64 \%$ (46/55), which is much higher than any reported sample types with negative smear tests to date. Four non-TB patients also produced positive outcomes, including one "low" and three "very low" positives. Only one patient had a distinct TB contact history, whereas no TB had been diagnosed for him before this data analysis.

Theoretically, the good performance of our study was highly dependent on the skill of our professionals for obtaining biopsy samples. The successful collection of tissue from the target lesion in the lung was also the key for XpertUltra evaluation [14]. Our study at least proved that even with a minute amount of lung lesion, Xpert-Ultra works well. However, we must trade off between the gain and disadvantage of biopsy sampling. Percutaneous lung biopsy is an invasive examination and often accompanies local pain and percutaneous emphysema, and sometimes it can cause local inflammation as well [19]. The expenditure and inaccessibility of biopsy under CT scan guidance limit its usage for patients who need it desperately. In our hospital, percutaneous lung biopsy is performed only when making a differential diagnosis for a patient with a lung lesion is urgently required, frequently when malignant disease is a major assumptive diagnosis. In this study, for each enrolled patient who had alung lesion, all the sputa, BALF and tissue collected by bronchoscopy produced negative outcomes for TB detection.

The additional benefit of Xpert-Ultra when identifying RIF resistance in the biopsy tissue is an impressive feature of this study [18]. Xpert assay is widely acknowledged for its capacity to identify RIF resistance within about $2 \mathrm{~h}$. Five RIF-resistant cases were identified among 36 patients with "low" to "high" levels of positive readouts. This fact reinforces the advantage of Xpert-Ultra in the rapid detection of RIF resistance in biopsy specimens. The high RIF-resistant rate $(13.89 \%, 5 / 36)$ among the patients with reliable resistance outcomes reflects the challenges of TB control in China. Above all, four out of these five RIF-resistant patients had no TB history. The transmission of drug-resistant $\mathrm{TB}$, especially multiple-drug-resistant (MDR) TB, needs more attention. Nevertheless, the "trace" outcome of Xpert-Ultra indicates that the $r p o B$ gene is not detectable; therefore, no resistance outcome can be acquired. Furthermore, the "very low" positive category is not reliable for determining its RIF-resistance outcome [8]. Due to the small amount of biopsy tissue, we obtained many "very low" positives (14 out of 49 positive specimens from TB patients, 17 out of all 53 positive outcomes in this study). Overall, about two thirds of the Xpert-Ultra-positive patients can benefit from the rapid RIF-resistance detection capacity of the assay. 
Notably, four enrolled patients were finally diagnosed with lung cancer with TB comorbidity. This fact highlights that although XpertUltra can successfully detect $\mathrm{TB}$, pathologic examination is still necessary for the diagnosis of other diseases. Xpert-Ultra positive results cannot be used to rule out lung cancer.

There are some limitations to this study. First, it was a single-center study; therefore, the findings and conclusions of the study need further validation in other centers. Second, an indeterminate outcome of the Xpert-Ultra assay, although it rarely happens, hurts the value of this invasive sampling method. Third, there is an argument about whether the reference standard test with biopsy tissue can also yield good sensitivity for TB diagnosis. This might be right to some extent. However, the low sensitivity of AFB detection with tissue and non-specific characteristics of non-typical granuloma image is always a concern for histopathologic examination. Nowadays, molecular tests are being increasingly applied for tissue exanimation to compensate for the histopathologic examination. For culture-negative patients, especially when malignant disease cannot be excluded according to the available data of intensive investigations, percutaneous lung biopsy combined with Xpert-Ultra assay could be a feasible choice.

\section{CONCLUSION}

Percutaneous lung biopsy combined with Xpert-Ultra produced high sensitivity for culture-negative pulmonary TB patient diagnosis in this study, supporting its usage for the differential diagnosis of lung disease and rapid MDR detection. "Trace" outcome using the lung biopsy specimen might not be a reliable positive category for $\mathrm{TB}$ diagnosis in a high-burden country.

\section{ACKNOWLEDGEMENTS}

We thank the participants of the study.
Funding. This study was supported by research funding from the Infectious Diseases Special Project, Ministry of Health of China (2018ZX10302301-004, 2018ZX10302302-004005), the Natural Science Fund of China (81802057, 81672065), Municipal Science \& Technology Commission (Z181100001718092), Beijing Municipal Administration of Hospitals Clinical Medicine Development of Special Funding Support (ZYLX201824) and Beijing Municipal Administration of Hospitals' Ascent Plan (DFL20181602). The Rapid Service Fees were funded by the authors.

Authorship. All authors meet the International Committee of Medical Journal Editors (ICMJE) criteria for authorship for this article, take responsibility for the integrity of the work as a whole, and have given their approval for this version to be published.

Disclosures. Zhen Zhou, Chenghai Li, Rui Zhu, Dongpo Wang, Tao Liu, Junnan Jia, Fen Wang,Liping Zhao, Lingling Dong, Xia Yu and Hairong Huang declare that they have no conflict of interest.

Compliance with Ethics Guidelines. This study has been approved by the Ethics Committee of the Beijing Chest Hospital, Capital Medical University. The study was conducted according to the Helsinki Declaration of 1964, as revised in 2013, concerning human and animal rights, and Springer's policy concerning informed consent has been followed.

Data Availability. The datasets generated during and analyzed during the current study are available from the corresponding author on reasonable request.

Open Access. This article is licensed under a Creative Commons Attribution-NonCommercial 4.0 International License, which permits any non-commercial use, sharing, adaptation, distribution and reproduction in any medium or format, as long as you give appropriate credit to the original author(s) and the source, provide a link to the Creative Commons licence, and indicate if changes were made. The images or 
other third party material in this article are included in the article's Creative Commons licence, unless indicated otherwise in a credit line to the material. If material is not included in the article's Creative Commons licence and your intended use is not permitted by statutory regulation or exceeds the permitted use, you will need to obtain permission directly from the copyright holder. To view a copy of this licence, visit http://creativecommons.org/licenses/by$\mathrm{nc} / 4.0 /$.

\section{REFERENCES}

1. World Health Organization. Global tuberculosis report 2019. https://www.who.int/tb/publications/ global_report/en/. 2019.Accessed 17 Oct 2019.

2. Feng RM, Zong YN, Cao SM, et al. Current cancer situation in China: good or bad news from the 2018 Global Cancer Statistics? Cancer Commun (Lond). 2019;39:22.

3. Kang F, Wang S, Tian F, et al. Comparing the diagnostic potential of $68 \mathrm{Ga}$-Alfatide II and $18 \mathrm{~F}-$ FDG in differentiating between non-small cell lung cancer and tuberculosis. J Nucl Med. 2016;57: 672-7.

4. Seijo LM, Peled N, Ajona D, et al. Biomarkers in lung cancer screening: achievements, promises, and challenges. J Thorac Oncol. 2019;14:343-57.

5. Kothary N, Lock L, Sze DY, et al. Computed tomography-guided percutaneous needle biopsy of pulmonary nodules: impact of nodule size on diagnostic accuracy. Clin Lung Cancer. 2009;10: 360-3.

6. Chakravorty S, Simmons AM, Rowneki M, et al. The new xpert MTB/RIF ultra: improving detection of mycobacterium tuberculosis and resistance to rifampin in an assay suitable for point-of-care testing. mBio. 2017;8(4):e00812-7.

7. Sun Q, Wang S, Dong W, et al. Diagnostic value of Xpert MTB/RIF Ultra for osteoarticular tuberculosis. J Infect. 2019;79:153-8.

8. Ocheretina O, Byrt E, Mabou MM, et al. False-positive rifampin resistant results with Xpert MTB/RIF version 4 assay in clinical samples with a low bacterial load. Diagn Microbiol Infect Dis. 2016;85: $53-5$.
9. Bates M, O'Grady J, Maeurer M, et al. Assessment of the Xpert MTB/RIF assay for diagnosis of tuberculosis with gastric lavage aspirates in children in subSaharan Africa: a prospective descriptive study. Lancet Infect Dis. 2013;13:36-42.

10. Bobbio F, Di Gennaro F, Marotta C, et al. Focused ultrasound to diagnose HIV-associated tuberculosis (FASH) in the extremely resource-limited setting of South Sudan: a cross-sectional study. BMJ Open. 2019;9:e027179.

11. Di Gennaro F, Pisani L, Veronese N, et al. Potential diagnostic properties of chest ultrasound in thoracic tuberculosis-a systematic review. Int J Environ Res Public Health. 2018;15(10):2235.

12. Bahr NC, Nuwagira E, Evans EE, et al. Diagnostic accuracy of Xpert MTB/RIF Ultra for tuberculous meningitis in HIV-infected adults: a prospective cohort study. Lancet Infect Dis. 2018;18:68-75.

13. Wang G, Wang S, Yang X, et al. Accuracy of Xpert MTB/RIF ultra for the diagnosis of pleural TB in a multicenter cohort study. Chest. 2020;157:268-75.

14. Yao X, Gomes MM, Tsao MS, et al. Fine-needle aspiration biopsy versus core-needle biopsy in diagnosing lung cancer: a systematic review. Curr Oncol. 2012;19:e16-27.

15. Kendall EA, Schumacher SG, Denkinger CM, et al. Estimated clinical impact of the Xpert MTB/RIF Ultra cartridge for diagnosis of pulmonary tuberculosis: a modeling study. PLoS Med. 2017;14: e1002472.

16. Wang G, Wang S, Jiang G, et al. Xpert MTB/RIF Ultra improved the diagnosis of paucibacillary tuberculosis: a prospective cohort study. J Infect. 2019;78:311-6.

17. Dorman SE, Schumacher SG, Alland D, et al. Xpert MTB/RIF Ultra for detection of Mycobacterium tuberculosis and rifampicin resistance: a prospective multicentre diagnostic accuracy study. Lancet Infect Dis. 2018;18:76-84.

18. World Health Organization. WHO meeting report of a technical expert consultation: non-inferiority analysis of Xpert MTB/RIF ultra compared to Xpert MTB/RIF. Geneva: World Health Organization; 2017.

19. Bhatt KM, Tandon YK, Graham R, et al. Electromagnetic navigational bronchoscopy versus CTguided percutaneous sampling of peripheral indeterminate pulmonary nodules: a cohort study. Radiology. 2018;286:1052-61. 